\title{
Quantitative Analysis of the Full Bitcoin Transaction Graph
}

\author{
Dorit Ron and Adi Shamir \\ Department of Computer Science and Applied Mathematics, \\ The Weizmann Institute of Science, Israel \\ \{dorit.ron, adi.shamir\}@weizmann.ac.il
}

\begin{abstract}
The Bitcoin scheme is a rare example of a large scale global payment system in which all the transactions are publicly accessible (but in an anonymous way). We downloaded the full history of this scheme, and analyzed many statistical properties of its associated transaction graph. In this paper we answer for the first time a variety of interesting questions about the typical behavior of users, how they acquire and how they spend their bitcoins, the balance of bitcoins they keep in their accounts, and how they move bitcoins between their various accounts in order to better protect their privacy. In addition, we isolated all the large transactions in the system, and discovered that almost all of them are closely related to a single large transaction that took place in November 2010 , even though the associated users apparently tried to hide this fact with many strange looking long chains and fork-merge structures in the transaction graph.
\end{abstract}

Keywords: bitcoin, digital coins, electronic cash, payment systems, transaction graphs, quantitative analysis

\section{Introduction}

Bitcoins are digital coins which are not issued by any government, bank, or organization, and rely on cryptographic protocols and a distributed network of users to mint, store, and transfer. The scheme was first suggested in 2008 by Satoshi Nakamoto [1], and became fully operational in January 2009. It had attracted a large number of users and a lot of media attention [2] [3] [4], but so far it was difficult to get precise answers to simple questions such as: How many different users are there in the system? How many bitcoins are typically kept in each account, and how does this balance vary over time? Are most bitcoins kept by a few large users? Do they keep their bitcoins in "saving accounts" or do they spend them immediately? How many users had large balances at some point in time? What is the size distribution of bitcoin transactions, and how many of them are micropayments?

In this paper we answer all these (and many additional) questions. We use the fact that all the transactions ever carried out in the Bitcoin system are available on the internet (in an anonymous way). On May $13^{\text {th }} 2012$ we downloaded the 
full public record of this system in one of its two major forms ${ }^{1}$, which consisted of about 180,000 HTML files. After parsing and processing these files, we built a graph of all the Bitcoin addresses and transactions up to that date. We then used the methodology described in the next section in order to try to identify which addresses are likely to belong to the same entity, and used this information to contract the transaction graph by merging such addresses, in order to get a more accurate picture of the full financial activity of each user. We then analyzed many statistical properties of both the original and the contracted transaction graphs (most of our statistical results were very similar for the two graphs, within a factor of 2). The most interesting and informative distributions we found are described in a series of tables. In addition, we isolated all the large $(\geq 50,000$ bitcoins) transactions which were ever recorded in the system, and analyzed how these amounts were accumulated and then spent. We discovered that almost all these large transactions were the descendants of a single large transaction involving 90,000 bitcoins which took place on November $8^{t h} 2010$, and that the subgraph of these transactions contains many strange looking chains and forkmerge structures, in which a large balance is either transferred within a few hours through hundreds of temporary intermediate accounts, or split into many small amounts which are sent to different accounts only in order to be recombined shortly afterwards into essentially the same amount in a new account.

There was one previous reported attempt [5] to download and analyze the full Bitcoin history, which also used the same methodology to try to contract all the addresses which are believed to belong to the same user. They created the graph of transactions on July $12^{\text {th }} 2011$, which was before the scheme really caught on. Thus, the total number of bitcoins participating in all the transactions in our graph is about three times larger than in their graph. In addition, we expect the transactions in our more mature graph to better represent typical use of the system, whereas their graph represents primarily the experiments run by early adopters. However, the biggest difference between our papers is that they were primarily interested in privacy issues, whereas we are primarily interested in the statistical properties of the Bitcoin transaction graph. Another analysis of the Bitcoin transaction graph was presented at the Chaos Computer Club Conference in Germany in December 2011 [6]. Again, they were primarily interested in how to defeat the anonymity of the network, but also included some interesting comments about the economic principles behind the scheme, the effect of lost coins on its operation, weaknesses in its protocols, and the general topological properties of this transaction graph.

The paper is organized as follows. In Section 2 we describe the Bitcoin scheme. In Section 3 we summarize the main statistical distributions we extracted from the downloaded transactions, which describe many interesting and even surprising properties of the scheme. Finally, in Section 4 we present the graph of the largest transactions and analyze its strange structure.

\footnotetext{
${ }^{1}$ We believe (but did not verify) that these two forms contain exactly the same information, and even if there are tiny differences they are likely to have only a negligible effect on our statistical results.
} 


\section{The Bitcoin Scheme}

Bitcoin is a decentralized electronic cash system using peer-to-peer networking to enable payments between parties without relying on mutual trust. It was first described in a paper by Satoshi Nakamoto (widely presumed to be a pseudonym) in 2008. Payments are made in bitcoins (BTC's), which are digital coins issued and transferred by the Bitcoin network. The data of all these transactions, after being validated with a proof-of-work system, is collected into what is called the block chain.

Participants begin using bitcoin by first acquiring a program called a Bitcoin wallet and one or more Bitcoin addresses. Bitcoin addresses are used for receiving bitcoins, in the same way that e-mail addresses are used for receiving e-mails. Even though Bitcoin is considered to be an experimental payment system, it is already deployed on a large scale (in the sense that the current value of all the coins issued so far exceeds 100,000,000 USD) and attracts a lot of media attention. Its proponents claim that it is the first truly global currency which does not discriminate its users based on citizenship or location, it is always running with no holidays, it is easy to secure with very low usage fees, it has no chargebacks, etc. On the other hand, its detractors claim that it is widely misused to buy illegal items and to launder large sums of money, and that it is too easy to steal bitcoins from wallets via cyber attacks.

Unlike fiat currency, which has been declared to be legal tender by a government despite the fact that it has no intrinsic value and is not backed by reserves, the Bitcoin scheme has no centralized issuing authority. The network is programmed to increase the money supply in a slowly increasing geometric series until the total number of bitcoins reaches an upper limit of about 21 million BTC's. Bitcoins are awarded to Bitcoin "miners" for solving increasingly difficult proof-of-work problems which confirm transactions and prevent doublespending. The network currently requires over one million times more work for confirming a block and receiving an award (currently 50 BTC's) than when the first blocks were confirmed.

The exchange rate of bitcoins has fluctuated widely over the years, from merely $\$ 0.01$ to over $\$ 30$ per BTC. Today (October 2012) it is worth a little over $\$ 12$ per BTC. The entire activity in the Bitcoin network is publicly available through the internet in two major forms, and the one we decided to download appears as a block chain, starting at block 0 [7] (created back on the $3^{\text {rd }}$ of January 2009). Each block reports on as little as a single transaction to as much as over a thousand transactions, and provides hyperlinks to other blocks and to other activities of each address.

Many users adopt the Bitcoin payment system for political and philosophical reasons. Each user can have an unbounded number of addresses (which are characterized by their public/private key pairs) owned by him. A transaction in bitcoins is a generalization of a regular bank transaction in the sense that it allows multiple sending addresses and multiple receiving addresses in the same transaction. It specifies how many bitcoins were taken from each sending address and how many bitcoins were credited to each receiving address, without the de- 
tails of who gave how much to whom. An address may receive bitcoins which are either newly minted or have a specific sending address. Another important difference between Bitcoin transactions and regular bank transactions is the notion of change, which is related to the fact that bitcoins are kept in (possibly fractional sized) chunks which have to be transferred in an all or nothing way. For example, a user can have three chunks of 10 bitcoins each. A transaction can spend 12.5 bitcoins by transferring the first full chunk plus 2.5 bitcoins from the second chunk, and then the 7.5 bitcoin change should be sent to a new address owned by the same user with new public and private keys. The user then has the option of either transferring the third chunk to the new address, or leaving it in the old address. In fact, it is considered good practice for a user to generate a new address, i.e., public-private key-pair, for every transaction even if this is not necessary. To better protect their identity, users are advised to take the following steps: they do not have to reveal any identifying information in connection with their addresses; they can repeatedly send varying fractions of their BTC's to themselves using multiple (newly generated) addresses; and/or they can use a trusted third-party in the form of a shared e-wallet to mix their transactions with those of other owners.

These operational and privacy policies of the Bitcoin scheme make it desirable for us to try to contract the transaction graph in order to get a more informative picture of the total assets and financial activities of users which are associated with many addresses, and to try to distinguish between "internal" and "external" transfers of bitcoins in it. Performing this contraction in a completely accurate way seems to be extremely difficult, but we can use the available data in order to try to find a good first approximation. Since many transactions have multiple sending addresses, we can make the reasonable assumption that all these addresses have the same owner. We then compute the transitive closure of this property over all the transactions. For example, if there is one transaction in which 1 and 2 are used as sending addresses, and another transaction in which 2 and 3 are used as sending addresses, we conclude that all three addresses are jointly owned. This can lead to two types of errors: We can underestimate the common ownership of some addresses because there was no evidence for it in the available data, and we can overestimate it if several users decided to pool their activities and to send a single transaction to which each one of them contributes some of the sending addresses. Discussions with several members of the Bitcoin community lead us to believe that at the moment there are likely to be very few overestimation errors of this type, but quite a few underestimation errors. For example, when we tried to use all the available transactions to merge the addresses of a particular large user, we were told that we managed to identify with our methodology only about one quarter of his real addresses. Note that the linkability of the addresses does not imply that the identity of the user becomes known. However, if we have any external information about the real ownership of any one of the merged addresses, we can get a fuller picture of the Bitcoin activity of that particular individual or organization. For example, since WikiLeaks publicly advertised one of its addresses when it asked for donations, we 
can estimate with our methodology that WikiLeaks owns at least 83 addresses, that it was involved in at least 1088 transactions, and that it had an accumulated income in all these addresses of 2605.25 BTC's.

We acquired the complete state of the Bitcoin transaction system on May $13^{\text {th }} 2012$, which contained all the transactions carried out in the system since its inception on January $3^{\text {rd }} 2009$ until that date. This required downloading 180,001 separate but linked HTML files, starting from block number 180,000 [8] and following the links backwards to the zeroth block initiating the system in January 2009. Each file was parsed in order to extract all the multisender/multireceiver transactions in it, and then the collection of transactions was encoded as a standard database on our local machine. We then ran a variant of a Union-Find graph algorithm [9] in order to find sets of addresses which are expected to belong to the same user. We merged all the nodes and combined all the transactions which can be associated with him (without eliminating the internal transfers, which become self loops in the new graph). We call the original transaction graph the address graph, and the contracted transaction graph the entity graph (we avoid using the word "owner" with its complex legal connotations since we do not really know who owns each address, and instead use the neutral word "entity" as our best approximation to the common owner of multiple addresses). All the statistics described in Section 3 are derived from both the address graph and the entity graph, as indicated in the tables. In most (but not all) cases, we expect the statistics to change monotonically as we move from the address graph to the entity graph and then to the (unknown) owner graph, since each entity is typically the union of several addresses which we managed to merge, and each real owner is typically the union of several entities that we failed to merge. For example, since the average balance of an address is 2.4 BTC's and the average balance of an entity is 3.7 , we can argue that the average balance of an owner is likely to be larger than 3.7 BTC's. This monotonicity can thus be used to provide plausible upper or lower bounds for the statistical properties of the real ownership graph, even though we do not know it.

\section{Statistics Calculated Over the Bitcoin Transaction Graph}

At the time we downloaded the graph there were 3,730,218 different public keys, each associated with a different address: $3,120,948$ of them were involved as senders in at least one transaction, while the additional 609,270 appear in the network only as receivers of BTC's. By running the Union-Find algorithm, we were able to associate the $3,120,948$ addresses with 1,851,544 different entities. Since the other 609,270 addresses were never used as senders, they could not be merged with any other addresses by the Union-Find algorithm, and thus they all remained as entities with a single address. By adding these singletons, we get a total of 2,460,814 entities, which implies that each one of them has on average about 1.5 addresses. However, there is a huge variance in this statistics, and in fact one entity is associated with 156,722 different addresses. By analyzing 
some of these addresses and following their transactions, it is easy to determine that this entity is Mt.Gox, which is the most popular Bitcoin Exchange site (responsible for almost $90 \%$ of all the exchange operations in the network). The full distribution of the number of addresses per entity is given in Table 1.

Table 1. The distribution of the number of addresses per entity

\begin{tabular}{ccc}
\hline Larger or equal to & Smaller than & Number of entities \\
\hline 1 & 2 & $2,214,186$ \\
2 & 10 & 234,015 \\
10 & 100 & 12,026 \\
100 & 500 & 499 \\
500 & 1,000 & 35 \\
1,000 & 5,000 & 41 \\
5,000 & 10,000 & 5 \\
10,000 & 50,000 & 5 \\
50,000 & 100,000 & 1 \\
100,000 & & 1 \\
\hline
\end{tabular}

In our reduced entity graph, each $m$-to- $n$ transaction has a single sender (since the $m$ sending addresses necessarily belong to the same entity) and at most $n$ receivers. It can thus be decomposed into at most $n$ different transactions from the single entity associated with the $m$ senders to the entities associated with the $n$ receivers. In case some of the receiving addresses are identified as belonging to the same entity, their amounts are accumulated to create a single common transaction, and if some of the receivers are identified with the single sender, we create a single self loop with the combined amounts. The resulting entity graph has $7,134,836$ single sender and single receiver transactions, out of which 814,044 (about 11\%) involve Deepbit (the largest Bitcoin mining pool), and 477,526 (about 7\%) involve Mt.Gox. About $10 \%$ of the transactions are self loops. The entity graph is not connected as it is composed of 133,742 different connected components, many of size one. For instance, there are as many as 43,710 components (about 33\%) consisting of a single address which are used only for accepting (one or several batches of) freshly minted bitcoins, and which have never participated in any incoming or outgoing transactions. Note that the address graph has a larger number of 13,734,847 transactions of lower values, since a single transaction with 2 sending addresses and 3 receiving addresses is represented in the address graph as 6 single-sender and single-receiver transactions.

There are many types of statistics and graphs about the Bitcoin network which can be readily downloaded from the internet [10] [11]. However, these types of statistics tend to describe some global property of the network over time, such as the number of daily transactions, their total volume, the number of bitcoins minted so far, and the exchange rate between bitcoins and US dollars. We can 
go much further than that, since the entire transaction graph can be used to determine the financial history of each entity including all of its sending/receiving activities along with the daily balance of bitcoins in its various addresses and how they vary over time. Having this entity graph at hand enables us to study various statistical properties of the network, which are not easy to determine by following a small number of online links in the Blockexplorer representation of the Bitcoin network. In the rest of this section, we describe some of our findings so far.

Here is our first surprising discovery, which is related to the question of whether most bitcoins are stored or spent. The total number of BTC's in the system is linear in the number of blocks. Each block is associated with the generation of 50 new BTC's and thus there are 9,000,050 BTC's in our address graph (generated from the 180,001 blocks between block number zero and block number 180,000). If we sum up the amounts accumulated at the 609,270 addresses which only receive and never send any BTC's, we see that they contain $7,019,100$ BTC's, which are almost $78 \%$ of all existing BTC's. Due to the way bitcoins can be repeatedly moved to fresh addresses, some of which can be very recent, we can not claim that all these bitcoins are out of circulation. However, $76.5 \%$ of these $78 \%$ (i.e., $59.7 \%$ of all the coins in the system) are "old coins", defined as bitcoins received at some address more than three months before the cut off date (May $13^{\text {th }} 2012$ ), which were not followed by any outgoing transactions from that address after they were received. One can also argue that very old dormant bitcoins were simply abandoned or lost by users who experimented with the system in its early days, when it was very difficult to buy anything or to exchange bitcoins into dollars. To be even more cautious with our estimation of dormant bitcoins, we decided to ignore all the transactions which took place prior to July $18^{\text {th }} 2010$, when Mt.Gox started its exchange and price quoting services. The sum of the balances of all the addresses which have not been active since that date is $1,657,480$ bitcoins. Clearly, by considering all these bitcoins as "lost" rather than "hoarded" we are underestimating the number of bitcoins which are kept dormant in "saving accounts". By ignoring these very old bitcoins and repeating the same calculation, we found that $73 \%$ of all the remaining BTC's were accumulated at addresses which only receive and never send bitcoins, and that $70 \%$ of these $73 \%$ (i.e., $51 \%$ ) are dormant bitcoins in the sense that they were received more than three months before our cutoff date but after it became easy to exchange them. If instead of summing the transaction values we sum the final balances of all the addresses that were active after July $18^{\text {th }} 2010$ but became inactive in the last three months, we get that $55 \%$ of all coins in the system are dormant in this sense. This is strong evidence that the majority of bitcoins are not circulating in the system, and since it is based on the address rather than the entity graph, this conclusion is not affected by possible inaccuracies in the way we associate addresses with users. Note that the total number of bitcoins participating in all the transactions since the establishment of the system (except for the actual minting operations) is 423,287,950 BTC's, 
and thus each coin which is in circulation had to be moved a large number of times to account for this total flow.

Another interesting finding is that the total number of bitcoins received by most entities and addresses is negligible. In the rest of this section, we use unparenthesized numbers to indicate values derived from the entity graph, and parenthesized numbers to indicate values derived from the address graph. For example, as can be seen from Table 2, 36\% of all entities (and $40 \%$ of all addresses) received fewer than one BTC, currently worth about 12 USD, throughout their lifetime, $52 \%$ (59\%) received fewer than 10 BTC's and $88 \%$ (91\%) fewer than 100. At the other end of the distribution there are only four entities (and one address) which received over 800,000 BTC's, and 80 entities (129 addresses) which received over 400,000.

Table 2. The distribution of the accumulated incoming BTC's per entity and per address

\begin{tabular}{cccc}
\hline Larger or equal to & Smaller than & Number of entities & Number of addresses \\
\hline 0 & 1 & 893,763 & $1,497,451$ \\
1 & 10 & 389,302 & 698,132 \\
10 & 100 & 881,273 & $1,206,209$ \\
100 & 1,000 & 255,826 & 285,820 \\
1,000 & 10,000 & 36,713 & 38,484 \\
10,000 & 50,000 & 3,593 & 3,723 \\
50,000 & 100,000 & 181 & 190 \\
100,000 & 200,000 & 55 & 50 \\
200,000 & 400,000 & 30 & 29 \\
400,000 & 800,000 & 76 & 129 \\
800,000 & & 4 & 1 \\
\hline
\end{tabular}

Similarly, as can be seen in Table 3 the current (on May $13^{\text {th }}$ 2012) balance of almost $97 \%(98 \%)$ of all entities (addresses) was less than 10 BTC's. This number decreases to $88 \%$ (91\%) if instead of looking at one specific moment, we look at the maximal balance ever seen throughout an entity's (address'es) lifetime. This statistics is summarized in Table 4 . In addition, it can be seen that there are only 78 entities (70 addresses) with current balance larger than 10,000 BTC's. This number grows to $3,812(3,876)$ when looking at the maximal balance ever seen.

Another measure that may indicate the level of activity of an entity (address) is the number of transactions it has been involved with. Its distribution is presented in Table 5. It is remarkable that 97\% (93\%) of all entities (addresses) had fewer than 10 transactions each, while 75 entities (80 addresses) use the network very often and are affiliated with at least 5,000 transactions.

We have also calculated the distribution of the size of the transactions in the two graphs as summarized in Table 6 . Again, it is evident that many transactions are very small, and $28 \%(47 \%)$ are smaller than 0.1 BTC each. The Bitcoin 
Table 3. The distribution of the current (on May $13^{\text {th }}$ 2012) balance of BTC's per entity and per address

\begin{tabular}{cccc}
\hline Larger or equal to & Smaller than & Number of entities & Number of addresses \\
\hline 0 & 0.01 & $2,097,245$ & $3,399,539$ \\
0.01 & 0.1 & 192,931 & 152,890 \\
0.1 & 10 & 95,396 & 101,186 \\
10 & 100 & 67,579 & 68,907 \\
100 & 1,000 & 6,746 & 6,778 \\
1,000 & 10,000 & 841 & 848 \\
10,000 & 50,000 & 71 & 65 \\
50,000 & 100,000 & 5 & 3 \\
100,000 & 200,000 & 1 & 1 \\
200,000 & 400,000 & 1 & 1 \\
400,000 & & 0 & 0 \\
\hline
\end{tabular}

Table 4. The distribution of the maximal balance of BTC's ever seen per entity and per address

\begin{tabular}{cccc}
\hline Larger or equal to & Smaller than & Number of entities & Number of addresses \\
\hline 0 & 0.1 & 547,763 & $1,063,876$ \\
0.1 & 10 & 668,247 & $1,160,170$ \\
10 & 100 & 945,083 & $1,188,596$ \\
100 & 1,000 & 259,142 & 276,613 \\
1,000 & 10,000 & 36,769 & 37,087 \\
10,000 & 50,000 & 3,513 & 3,521 \\
50,000 & 100,000 & 163 & 159 \\
100,000 & 200,000 & 40 & 41 \\
200,000 & 400,000 & 26 & 26 \\
400,000 & 500,000 & 68 & 129 \\
500,000 & & 2 & 0 \\
\hline
\end{tabular}

Table 5. The distribution of the number of transactions per entity and per address

\begin{tabular}{cccc}
\hline Larger or equal to & Smaller than & Number of entities & Number of addresses \\
\hline 1 & 2 & 557,783 & 495,773 \\
2 & 4 & $1,615,899$ & $2,197,836$ \\
4 & 10 & 222,433 & 780,433 \\
10 & 100 & 55,875 & 228,275 \\
100 & 1,000 & 8,464 & 26,789 \\
1,000 & 5,000 & 287 & 1,032 \\
5,000 & 10,000 & 35 & 51 \\
10,000 & 100,000 & 32 & 24 \\
100,000 & 500,000 & 7 & 3 \\
500,000 & & 1 & 2 \\
\hline
\end{tabular}


scheme actually enables sending micro transactions, which are of the order of $10^{-8}$ BTC (this is the smallest fraction into which a BTC can be broken, and is called a satoshi). When we also consider midsize amounts, we see that $73 \%$ $(84 \%)$ of the transactions involve fewer than 10 BTC's. On the other hand, large transactions are rare at Bitcoin: there are only 364 (340) transactions larger than 50,000 BTC's. We have carefully inspected all these large transactions and describe our findings in the next section.

Table 6. The distribution of the size of the transactions in the Bitcoin scheme

\begin{tabular}{cccc}
\hline Larger or equal to Smaller than & $\begin{array}{c}\text { Number of transactions } \\
\text { in the graph of entities }\end{array}$ & $\begin{array}{c}\text { Number of transactions } \\
\text { in }\end{array}$ \\
\hline 0 & 0.001 & 381,846 & $2,315,582$ \\
0.001 & 0.1 & $1,647,087$ & $4,127,192$ \\
0.1 & 1 & $1,553,766$ & $2,930,867$ \\
1 & 10 & $1,628,485$ & $2,230,077$ \\
10 & 50 & $1,071,199$ & $1,219,401$ \\
50 & 100 & 490,392 & 574,003 \\
100 & 500 & 283,152 & 262,251 \\
500 & 5,000 & 70,427 & 67,338 \\
5,000 & 20,000 & 6,309 & 6,000 \\
20,000 & 50,000 & 1,809 & 1,796 \\
50,000 & & 364 & 340 \\
\hline
\end{tabular}

It is interesting to investigate the most active entities in the Bitcoin system, those who have either maximal incoming BTC's or maximal number of transactions. 19 such entities are shown in Table 7 sorted in descending order of the number of accumulated incoming BTC's shown in the third column. The leftmost column associates the entities with letters between $\mathrm{A}$ to $\mathrm{S}$ out of which three are identified: B is Mt.Gox, G is Instawallet and L is Deepbit. Eight additional entities: F, H, J, M, N, O, P, and Q are pointed out in the graph of the largest transactions (Fig. 1) which is presented in the next section. The second column gives the number of addresses merged into each entity. The fourth column presents the number of transactions the entity is involved with.

Table 7 shows that Mt.Gox has the maximal number of addresses, but not the largest accumulated incoming BTC's nor the largest number of transactions. Entity A in the first row of Table 7 owns the next largest number of addresses, about $50 \%$ of those of Mt.Gox's, but received 31\% more BTC's than Mt.Gox. Deepbit had sent $70 \%$ more transactions than Mt.Gox. It is interesting to realize that the number of addresses of 13 of these entities is a fifth or more of the number of transactions they have executed, which may indicate that each address is indeed used for just a few transactions. It is also clear that six out of the 19 entities in the table have each sent fewer than 30 transactions with a total volume of more than 400,000 BTC's. Since these entities were using large transactions, we were able to isolate them and to follow the flow of their trans- 
Table 7. The list of most active entities in Bitcoin, which have either maximal incoming BTC's or maximal number of transactions. Some of the letters in the leftmost column: F, H, J, M, N, O, P and Q refer to the red letters in Fig. 1 pointing these entities out.

\begin{tabular}{cccc}
\hline Entity ID & $\begin{array}{c}\text { Number of } \\
\text { Addresses }\end{array}$ & $\begin{array}{c}\text { Accumulated } \\
\text { Incoming BTC's }\end{array}$ & $\begin{array}{c}\text { Number of } \\
\text { Transactions }\end{array}$ \\
\hline A & 78,251 & $2,886,650$ & 246,012 \\
B (Mt.Gox) & 156,722 & $2,206,170$ & 477,526 \\
C & 13,289 & 941,013 & 77,525 \\
D & 12,520 & 867,996 & 48,347 \\
E & 191 & 692,864 & 1,353 \\
F & 12 & 660,000 & 23 \\
G (Instawallet) & 23,649 & 633,606 & 92,593 \\
H & 9 & 580,000 & 59 \\
I & 10,561 & 514,066 & 49,550 \\
J & 4 & 500,021 & 6 \\
K & 134 & 479,254 & 1,039 \\
L (Deepbit) & 2 & 452,929 & 814,044 \\
M & 9 & 442,000 & 10 \\
N & 128 & 432,161 & 137 \\
O & 10 & 432,286 & 14 \\
P & 1 & 432,078 & 3 \\
Q & 14 & 430,490 & 23 \\
R & 2,124 & 321,866 & 300,486 \\
S & 1,037 & 20,308 & 197,334 \\
\hline
\end{tabular}


actions, see Section 4 below. On the other hand, entity A had never sent any large transactions and thus it has not been included in our graph of the largest transactions.

\section{The Graph of the Largest Transactions in Bitcoin}

We have identified and analyzed all the largest ( $\geq 50,000$ BTC's) transactions in the entity graph, (there were 364 such transactions as described in the last column of Table 6), and followed their flow. We started with the earliest such large transaction, the one of 90,000 BTC's made on November $8^{\text {th }} 2010$. By tracing each of the other 363 large transactions in this category, we were able to show that 348 were actual successors of this initial transaction. The resulting directed graph is depicted in Fig. 1. This graph reveals several characteristic behaviors of the flow in the Bitcoin transaction graph: long consecutive chains of transactions, fork-merge patterns that may include self loops, setting aside BTC's and final distribution of large sums via a binary tree-like structure.

Long Chains. A common prominent practice of Bitcoin users is to create chains of consecutive transactions. Some of these chains can be explained by the change mechanism in which small payments are accompanied by the creation of a new address, into which the user transfers the difference. Such chains can be found in Fig. 2, Fig. 4, Fig. 5 and Fig. 7, with lengths of 3, 15, 26, 80, 88 and 350 transactions. However, the behavior seen in Fig. 3 deviates significantly from this pattern, since the same amount of 5,000 bitcoins is repeatedly split off the main sum and put into accounts which have no additional transactions associated with them.

Fork-Merge Patterns and Self Loops. Another frequent scenario in Bitcoin is transferring a large number of BTC's from one address to another via several intermediate addresses, each receiving part of the entire amount and then sending it, mostly in full, to the same destination whether directly or via other mediators. Examples can be seen in Fig. 6, Fig. 8 and Fig. 9. A harder to follow fork-merge pattern is presented in Fig. 5: An entity is sending 90,000 BTC's to itself three times in self loops. Each time it splits it into different amounts, $76+14,72+18$ and $69+21$. It uses the same address for the small amounts and different addresses for the large amounts. Then it exchanges the entire 90,000 BTC's at Mt.Gox. Finally, the 90,000 BTC's are being transferred via a chain of 90 transactions using 90 different addresses (which may or may not belong to the same owner), where at each one of them 1,000 BTC's are sent back to the first entity, recombined into essentially the very first amount of 90,000 BTC's.

Keeping Bitcoins in "Saving Accounts". Another long chain of transactions from the beginning of March 2011 can be seen in Fig. 3. This chain is different from the above ones, since at 28 out of its 30 steps, it puts aside 5,000 BTC's in what seems to be "saving accounts". The accumulated sum of 140,000 BTC's has never been sent since. These bitcoins are an example of our discovery that most of the bitcoins are not circulating in the system. 
Binary Tree-Like Distributions. Often amounts of BTC's are distributed among many addresses by splitting it into two similar amounts at each step. This results in a binary tree-like structure as depicted in Fig. 10 and in Fig. 4.

\section{Conclusions}

The Bitcoin system is the best known and most widely used alternative payment scheme, but so far it was very difficult to get accurate information about how it is used in practice. In this paper we describe a large number of statistical properties of the Bitcoin transaction graph, which contains all the transactions which were carried out by all the users until May $13^{\text {th }}$ 2012. We discovered that most of the minted bitcoins remain dormant in addresses which had never participated in any outgoing transactions. We found out that there is a huge number of tiny transactions which move only a small fraction of a single bitcoin, but there are also hundreds of transactions which move more than 50,000 bitcoins. We analyzed all these large transactions by following in detail the way these sums were accumulated and the way they were dispersed, and realized that almost all these large transactions were descendants of a single transaction which was carried out in November 2010. Finally, we noted that the subgraph which contains these large transactions along with their neighborhood has many strange looking structures which could be an attempt to conceal the existence and relationship between these transactions, but such an attempt can be foiled by following the money trail in a sufficiently persistent way.

Acknowledgments. This research was supported by the Citi Foundation. We would like to thank Ronen Basri, Uriel Feige, Michal Irani, Robert Krauthgamer, Boaz Nadler, Moni Naor and David Peleg from the Computer Science and Applied Mathematics Department of the Weizmann Institute of Science for many interesting and informative discussions. We would also like to thank Aharon Friedman for his major help in acquiring and processing the Bitcoin data base. Finally, we would like to thank all the members of the Bitcoin community, and in particular Meni Rosenfeld and Stefan Richter, who sent us excellent comments, criticisms and suggestions. We revised the original version of the paper in order to respond to their input.

\section{References}

1. Nakamoto, S.: Bitcoin: A Peer-to-Peer Electronic Cash System, 2008.

2. Wallace, B.: The Rise and Fall of Bitcoin, Wired Magazine, 23 November 2011, http://www . wired.com/magazine/2011/11/mf_bitcoin/all/

3. NPR Staff: Silk Road: Not Your Father's Amazon.com, 12 June 2011, http://www . npr.org/2011/06/12/137138008/silk-road-not-your-fathers-amazon-com

4. Brett, W.: Senators seek crackdown on "Bitcoin" currency, Reuters, 8 Jun 2011, http://www.reuters.com/article/2011/06/08/ us-financial-bitcoins-idUSTRE7573T320110608 
5. Reid, F., Harrigan M.: An Analysis of Anonymity in the Bitcoin System, arXiv:1107.4524v2 [physics.soc-ph] 7 May 2012.

6. Hamacher, K., Katzenbeisser, S.: Bitcoin - An Analysis, 29 Dec 2011, http://www . youtube. com/watch?v=hlWyTqL1hFA

7. Bitcoin's block number 0, http://blockexplorer.com/b/0

8. Bitcoin's block number 180,000, http://blockexplorer.com/b/180000

9. Cormen, T.H., Leiserson, C.H., Rivest, R.L., Stein, C.: Introduction to Algorithms, Second Edition. MIT Press and McGrawHill, 2001. Chapter 21: Data structures for Disjoint Sets, pp. 498-524.

10. Forbes: Top 10 Bitcoin Statistics, http://www.forbes.com/sites/jonmatonis/ 2012/07/31/top-10-bitcoin-statistics/

11. Block chain: Bitcoin charts http://blockchain.info/charts

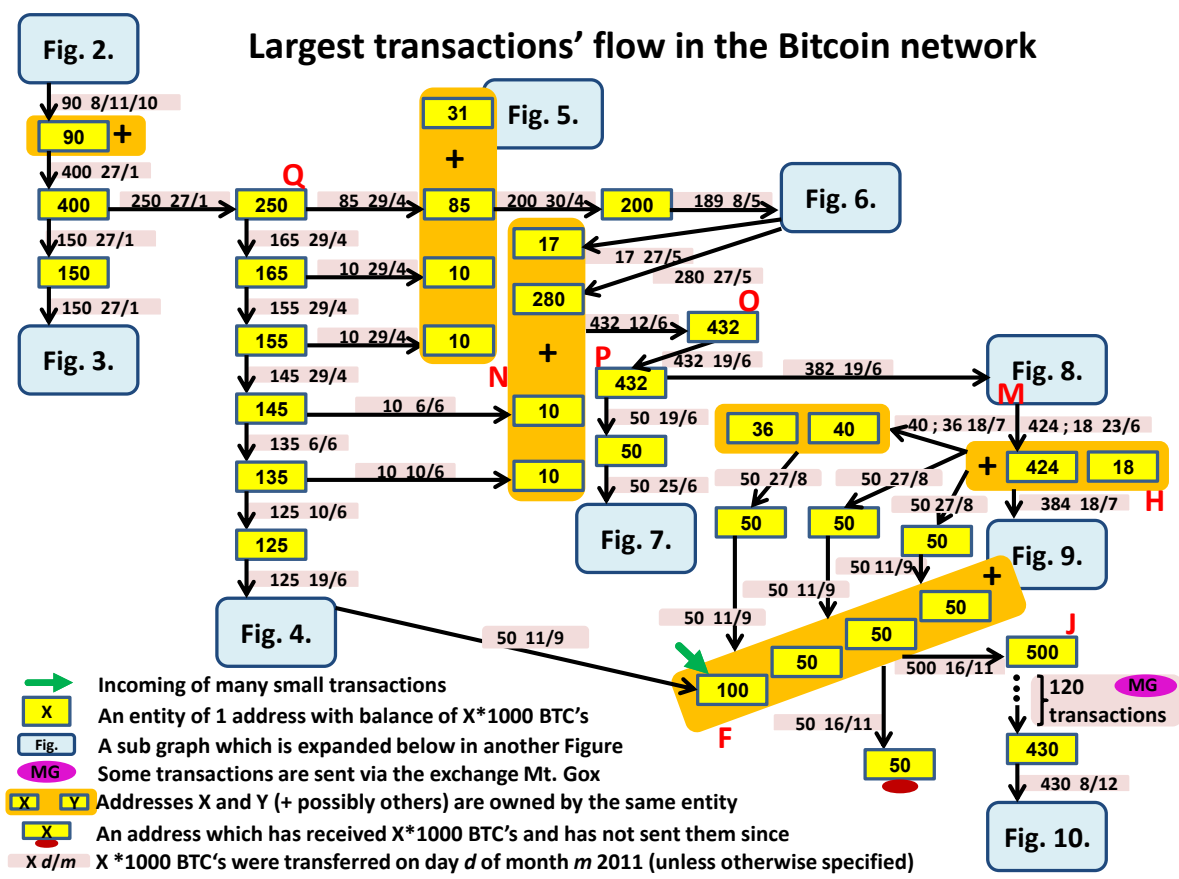

Fig. 1. The backbone of the graph of almost all largest transactions in the Bitcoin scheme (those which are larger than 50,000 BTC's). The red letters refer to some of the most active entities in Bitcoin as listed in Table 7. 


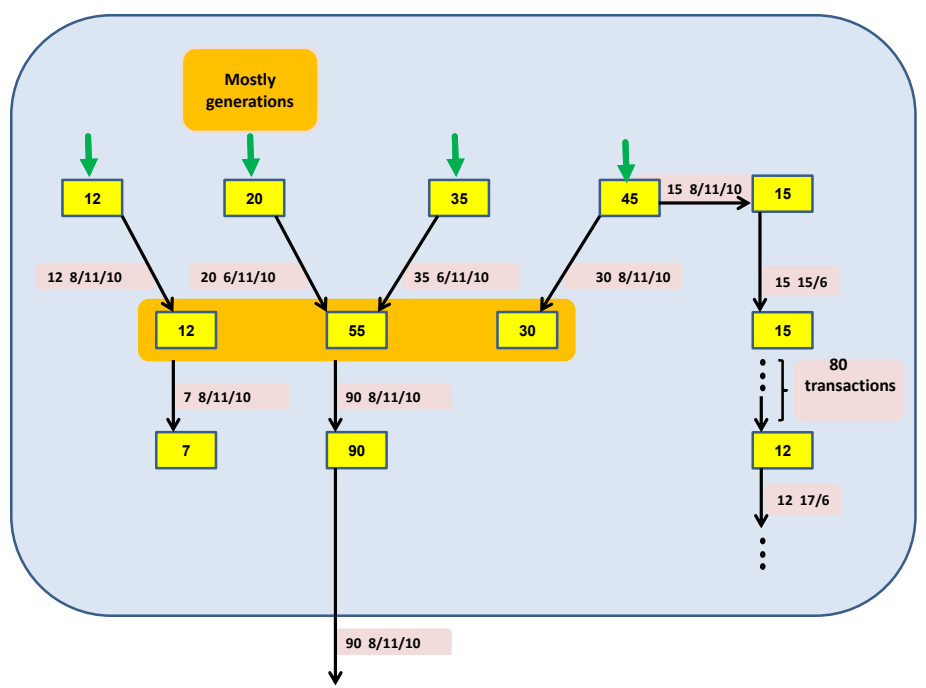

Fig. 2. A Sub graph of Fig. 1: A trace back of some flows of BTC's leading to the first large transaction of 90,000 BTC's on November $8^{\text {th }} 2010$.

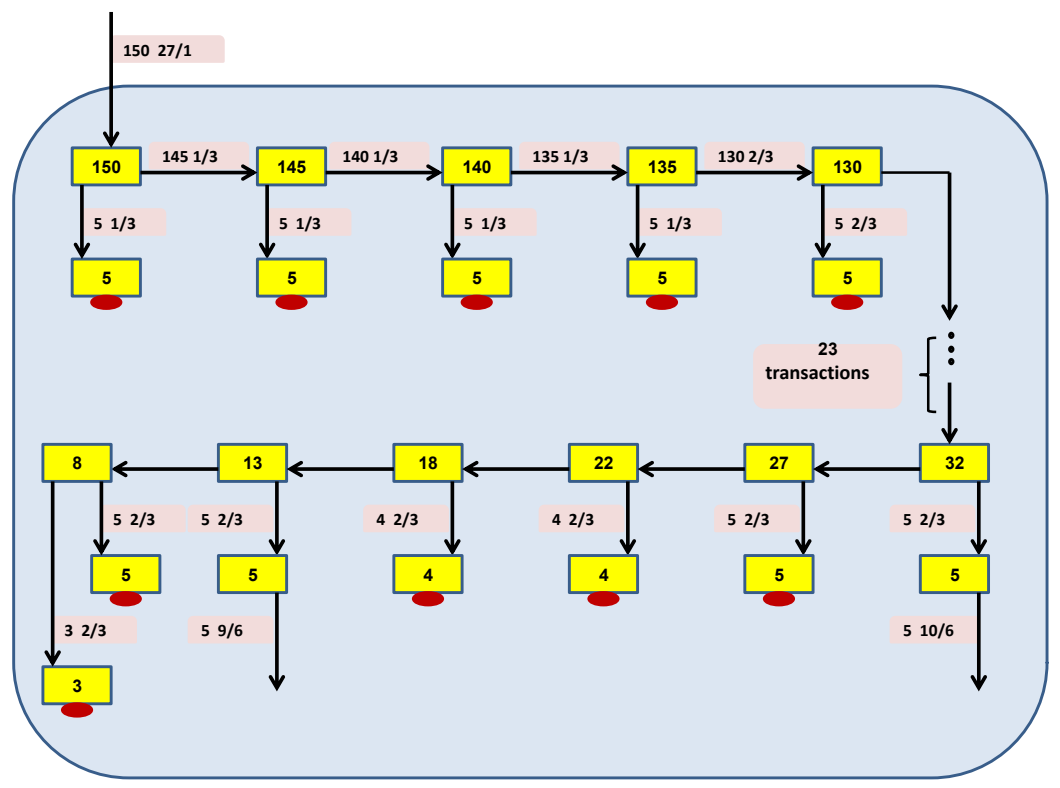

Fig. 3. A Sub graph of Fig. 1: A long chain of transactions where each address puts aside a small amount of BTC's. Those amounts sum up to 140,000 BTC's. 


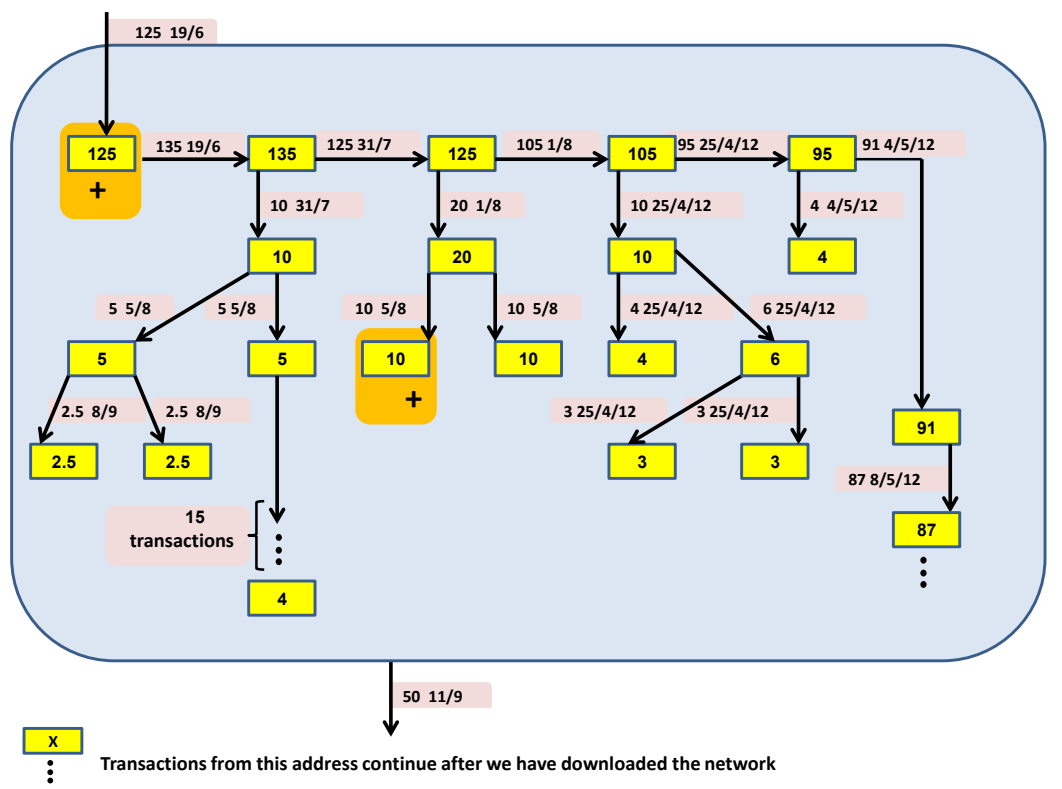

Fig. 4. A Sub graph of Fig. 1: A long chain of transactions where each address transfers most of its BTC's forward. The rest is distributed in a binary tree-like structure.

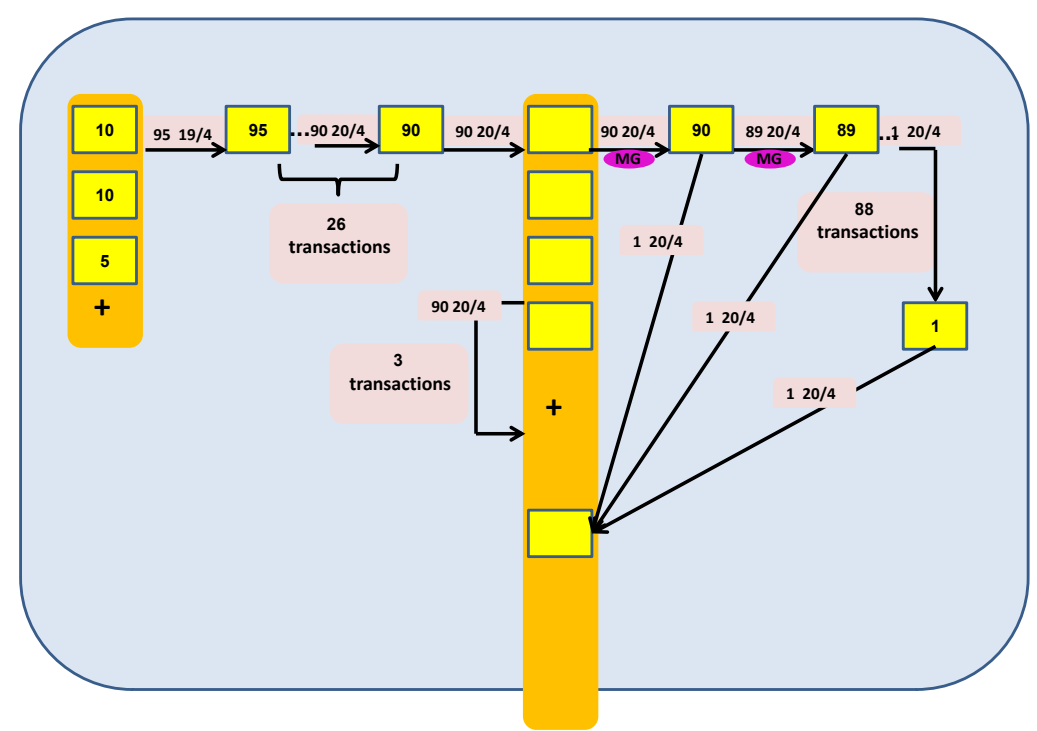

Fig. 5. A Sub graph of Fig. 1: An entity is sending 90,000 BTC's to itself in a self loop, then transfers it forward but gets it back via 90 transfers of 1,000 BTC's each, all carried out on the same day. 31,000 of it is then transferred forward. 


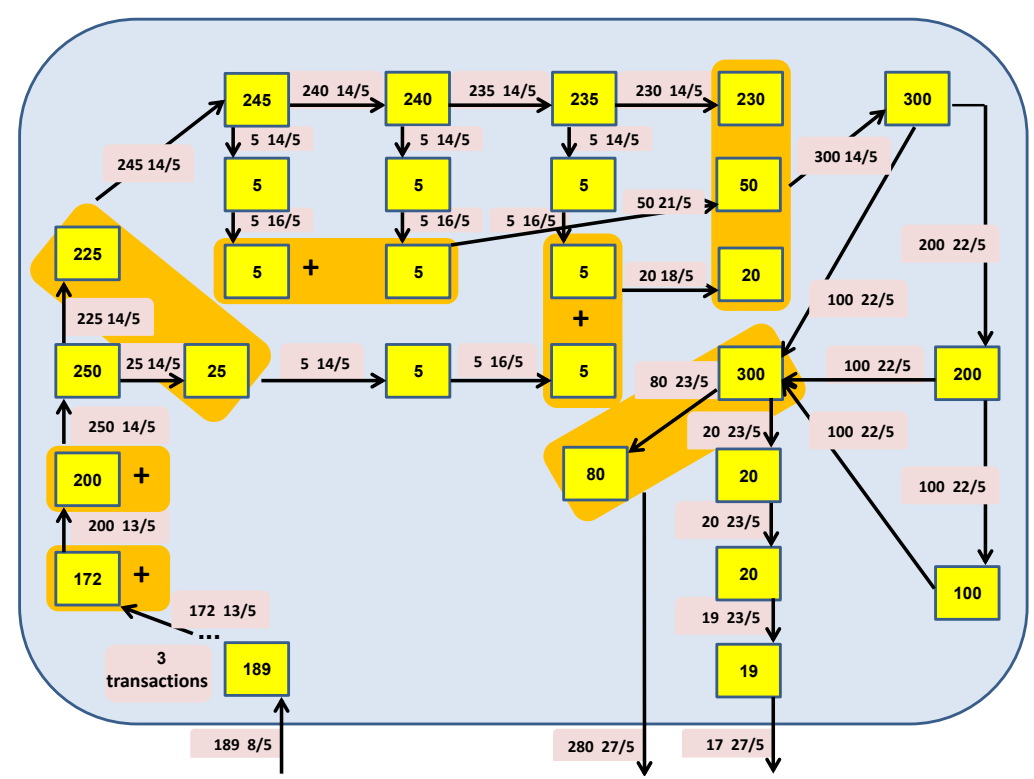

Fig. 6. A Sub graph of Fig. 1: Large amounts of BTC's are transferred from one address to another by sending parts of it to intermediate addresses, which are then being merged into the same destination.

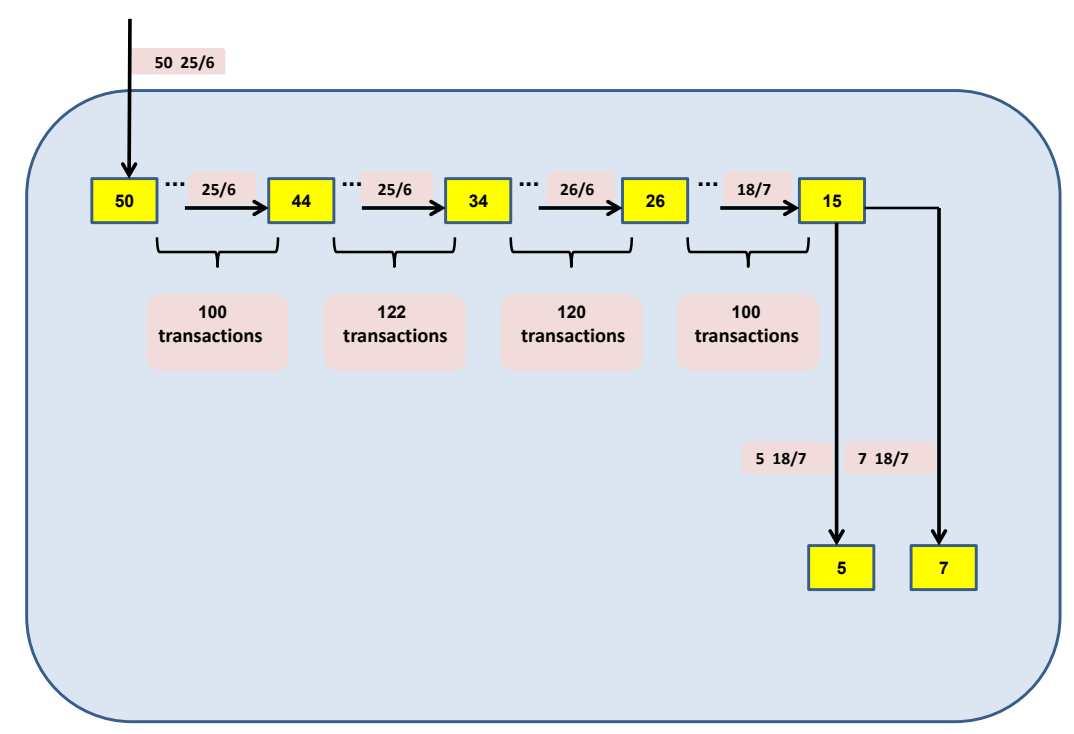

Fig. 7. A Sub graph of Fig. 1: Large amounts of BTC's are rapidly transferred in a very long chain of hundreds of transactions in a very short period of time. 


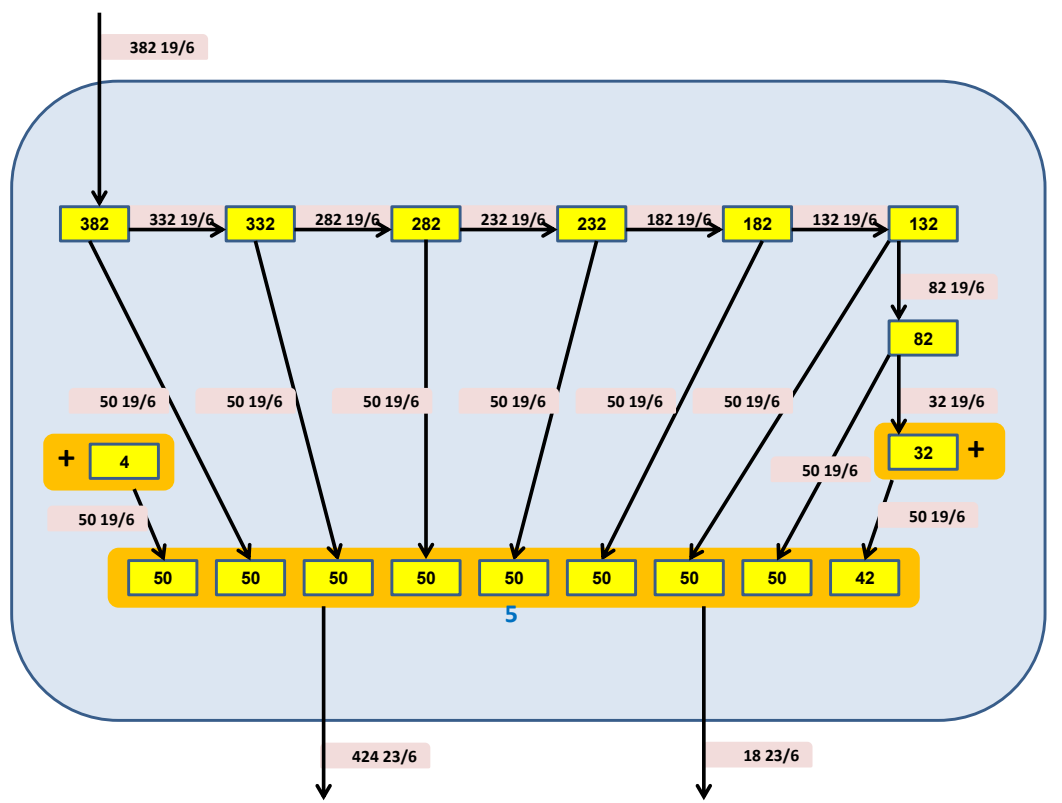

Fig. 8. A Sub graph of Fig. 1: A very large amount of BTC's is transferred by splitting it into equal amounts each directed to a different address belonging to the same entity, then most of the accumulated sums are transferred to a single receiver.

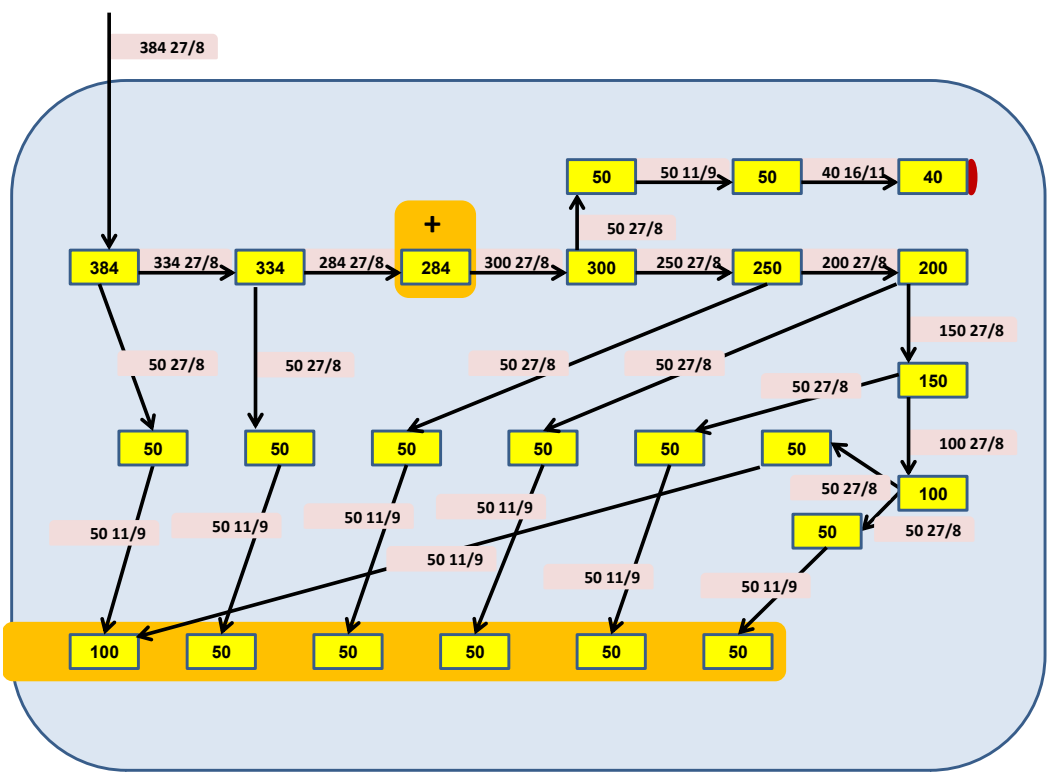

Fig. 9. A Sub graph of Fig. 1: A similar scenario as described in Fig. 8 but with more intermediate addresses. 


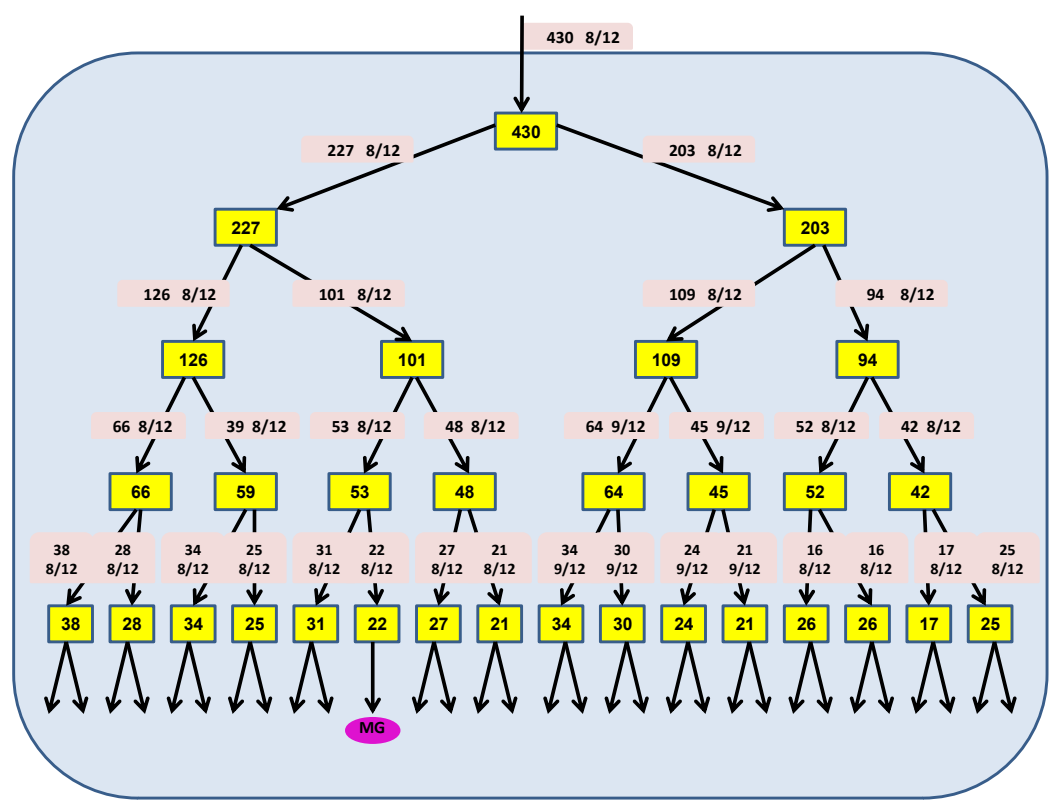

Fig. 10. A Sub graph of Fig. 1: The largest amount of transferred BTC's is finally distributed among many addresses via a binary tree-like structure. 